\title{
A rare case of tuberculous abscess causing airway obstruction in a paediatric patient in South-East Asia
}

\author{
Sharifah Intan Safuraa', Primuharsa Putra², Saraiza Abu Bakar², Dina Hashim¹, Zara \\ Case \\ Nasseri $^{1}$ \\ report \\ ${ }^{1}$ Department of Otorhinolaryngology- Head \& Neck Surgery, National University of
Malaysia Medical Centre, Kuala Lumpur, ${ }^{2}$ Ear, Nose, Throat-Head \& Neck Consultant
Clinic, KPJ Seremban Specialist Hospital/KPJ Healthcare University College, Negeri
Sembilan, ${ }^{3}$ Department of Otorhinolaryngology-Head \& Neck Surgery, Serdang Hospital,
Serdang, Selangor, Malaysia.
}

\begin{abstract}
A 6-year old girl, presented with symptoms of obstructive sleep apnoea. She was referred to our centre when she developed obstructive symptoms such as stridor and dysphagia a month later. She was found to have a right neck swelling with a posterior pharyngeal wall mass, which caused upper airway obstruction. A computerized-tomography scan of her neck showed a very narrowed upper airway with a retropharyngeal abscess without prevertebral or vertebral involvement. She had a challenging intubation and underwent an emergency intraoral incision and drainage. Pus that was drained revealed acid fast bacilli and histopathological examination showed necrotizing granulomatous inflammation, in favour of a tuberculous abscess. Post operatively, her symptoms resolved and her intraoral wound healed. She completed antituberculous treatment and has remained well at 6 months follow up. Our case illustrates how a cold retropharyngeal abscess can masquerade as obstructive sleep apnoea, with a compromised airway as it increases in size.
\end{abstract}

Key Words: Paediatric airway, retropharyngeal abscess, snoring, stridor, tuberculosis.

Received: 10 June 2018, Accepted: 15 May 2019

Corresponding Author: Zara Nasseri, MD, Department of Otorhinolaryngology- Head \& Neck Surgery, National University of Malaysia Medical Centre, Kuala Lumpur, Malaysia, Tel.: +60 39145 6045, E-mail: zaranasseriwork@gmail.com

ISSN: 2090-0740, July 2019 Vol.20, No.2

\section{INTRODUCTION}

Extrapulmonary tuberculosis (EPTB) constitutes up to $25 \%$ of cases of tuberculosis globally ${ }^{[1]}$. Locally, the reported cases of EPTB is approximately $15 \%$, and expectantly the proportion is double in immunocompromised patients ${ }^{[2]}$. Tuberculous lymphadenitis is the commonest form of EPTB, making up to $80 \%$ of cases ${ }^{[2]}$. These rarely lead to retropharyngeal abscesses. We report a case of a child who presented with symptoms of upper airway obstruction with a posterior pharyngeal wall mass, subsequently found to be caused by a tuberculous retropharyngeal abscess.

\section{MATERIALS AND METHODS}

Collected data from old case notes.

\section{CASE REPORT}

A 6-year old girl presented with a one month history of snoring, and progressively worsening dysphagia. A week prior to presentation, she developed right neck swelling with stridor at rest. She did not have cough, fever, tuberculous contact, trauma to the neck or foreign body ingestion. She had completed immunisation up to her age. There was no previous history of tuberculosis infection. Examination revealed a large right neck swelling and a prominent bulge along the entire visualised posterior pharyngeal wall, obstructing approximately $90 \%$ of the airway. A flexible nasopharyngolaryngoscopy was not performed and other examination was normal. She did not have any hepatosplenomegaly or generalized lymphadenopathy. Blood investigations showed her white cell count to be $13.8 \times 10^{9} / \mathrm{L}$ (predominantly neutrophils) and her erythrocyte sedimentation rate $46 / \mathrm{h}$. Computedtomography (CT) scan of the neck showed a well-defined and well-enhancing, hypodense lesion extending from base of skull to $\mathrm{C} 3$ vertebrae, causing severe narrowing of the naso-, oro- and hypopharynx, displacing the right internal carotid artery and internal jugular vein. However, the internal jugular vein was not thrombosed. There were multiple bilateral lymph nodes in the cervical region. There was no radiological evidence of prevertebral and vertebral involvement. Clinically and radiologically, the findings were consistent with the diagnosis of retropharyngeal abscess, therefore, prior to a formal incision and drainage under general anaesthesia, a needle aspiration was performed which yielded $4 \mathrm{mls}$ of pus. 
Otherwise, a direct laryngoscopy revealed normal findings. The pus was sent for analysis. Acid fast bacilli was seen on Ziehl-Neelsen staining. Bacterial culture was reported as negative after 6 weeks. Histopathological examination revealed a necrotizing granulomatous inflammation, highly suggestive of tuberculosis. Following a positive test for Ziehl-Neelsen staining from the intraoperative pus sample, a comprehensive tuberculosis workup was done that included a chest radiograph, which proved that there as no other foci of infection. Postoperative recovery was uneventful with complete resolution of the intraoral and right neck swelling. Her persistent low-grade fever resolved once she was started on oral anti-tuberculous regime of isoniazid, rifampicin, pyrazinamide and pyridoxine for six months, as per the World Health Association (WHO) and local tuberculosis guidelines. She has remained well with no recurrence of symptoms in the 6 months that followed completion of treatment.

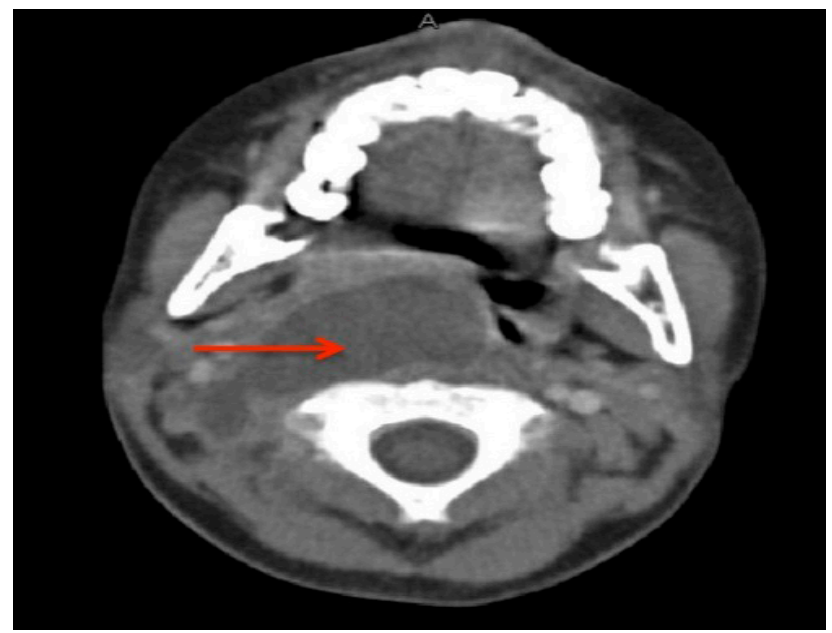

Fig. 1: CT axial image showing the well-defined and rimenhancing, hypodense lesion measuring $2.4 \times 5.7 \times 7.3 \mathrm{~cm}$ that caused narrowing of oropharynx, as shown by arrow.

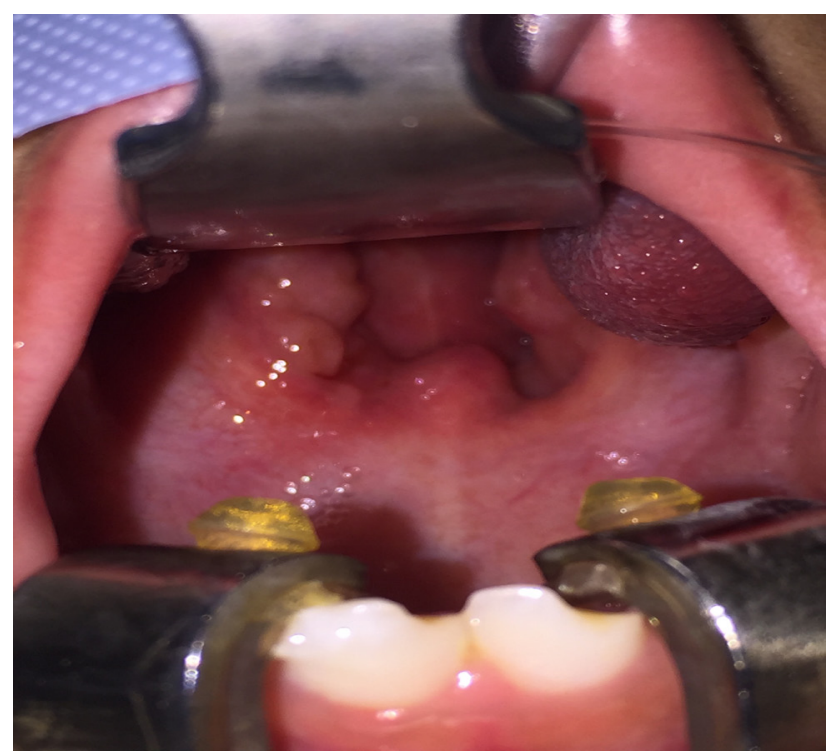

Fig. 2: Introral image showing bulging of the posterior oropharyngeal wall.

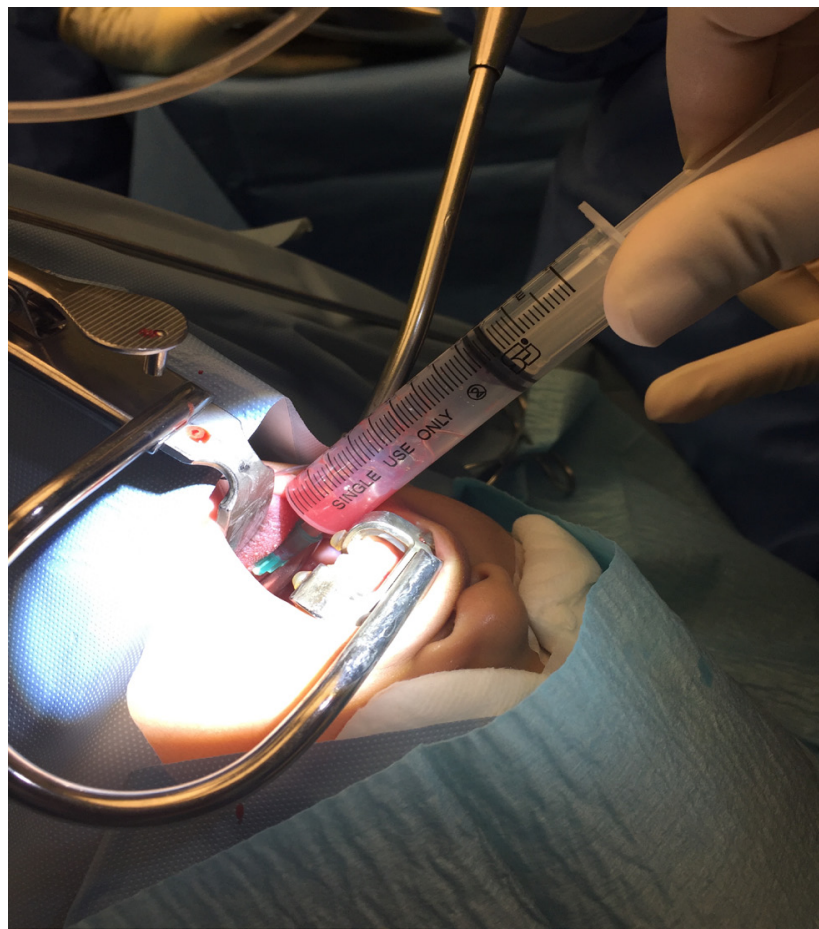

Fig. 3: Intraoperative needle aspiration, prior to the incision and drainage.

\section{DISCUSSION}

Tubercular retropharyngeal abscesses are rare in children ${ }^{[3]}$.

The retropharyngeal space is a potential space bounded anteriorly by the pharyngeal muscles and investing fascia and posteriorly by the prevertebral fascia. It extends from the skull base down to the mediastinum, and laterally bounded by the carotid sheath. It contains lymph nodes that drain from the oropharynx, teeth, maxillary sinus, and ears and regress by the age of 6 years ${ }^{[4]}$.

Retropharyngeal abscesses can be either acute or chronic. Acute retropharyngeal abscesses are commonly seen in children as a result of an upper respiratory tract infection. Retropharyngeal abscesses can also be associated with penetrating trauma and foreign body, all of which our case lacked ${ }^{[5,6]}$. Chronic retropharyngeal abscess is usually seen in adults and caused by spread of atypical infections, commonly tuberculous infections from the cervical spine, via the anterior longitudinal ligament ${ }^{[4]}$.

The probable route of spread of tuberculosis to the retropharyngeal space, in children, in the absence of cervical spine involvement, as in this case, is via the lymphatics, with subsequent suppuration of retropharyngeal lymph nodes and abscess formation ${ }^{[7]}$.

The common organism isolated in retropharyngeal abscess' in children are Group A -hemolytic Streptococcus. Less common organisms include Staphylococcus Aureus, 
Neisseria and Haemophilus and oropharyngeal anaerobes although often associated with mixed flora ${ }^{[8]}$.

Children with bacterial retropharyngeal abscess typically present with fever, neck pain, torticollis, neck stiffness, and presence of cervical lymphadenopathy ${ }^{[9]}$. Late presenting symptoms can include stridor, shortness of breath, dysphagia and sepsis. Aside from the presenting feature of stridor, in this case, the child had preceding symptoms that mimicked obstructive sleep apnoea. Apart from enlarged adenoids, she did not have poor school performance, short attention span, or enlarged tonsils, which are typical of children with obstructive sleep apnoea.

Diagnosis is difficult in children as symptoms are not specific and onset and progression of the disease can be gradual ${ }^{[10]}$. Compared to other lymph nodes in the head and neck region, retropharyngeal nodes are not palpable, which can lead to a rather late presentation of upper airway obtruction caused by bulging of the posterior pharyngeal wall. Furthermore, as occurred in this case, upper airway obstruction is an exceedingly rare presentation of tuberculous abscess ${ }^{[3]}$.

This is the seventh reported case of tuberculous retropharyngeal abscess in the paediatric age group in the world. Of the 6 reported, 4 of them presented with stridor, and 2 of the 4 did not have any cervical spine involvement, as in this case $^{[3,11-15]}$. An additional point to note in our case, is the child's initial presentation of obstructive sleep apnoea. In cases involving cervical spine tuberculosis as the cause of the retropharyngeal abscess, the presentation was longer, within months. In cases without cervical spine tuberculosis, the presentation was more acute ranging from days up to 2 weeks ${ }^{[3,11-16]}$.

A child in Korea, presented with a three day history of stridor, perioral cyanosis and refusing to feed, preceded by rhinorrhoea and sneezing ${ }^{[15]}$. In another reported case, in India, the child presented with progressive left neck swelling, stridor and low grade fever for two weeks ${ }^{[3]}$. In both cases, the children were fully immunised.

Early recognition of the symptoms and signs is paramount in preventing serious complications such as mediastinitis, upper airway obstruction and even spontaneous rupture of the abscess which can lead to tracheobronchial aspiration, all of which are associated with a high mortality rate ${ }^{[5]}$.

In cases of retropharyngeal abscess causing upper airway obstruction, difficult airway management is a major concern. Difficulties in tracheal intubation can be due to trismus, distorted laryngeal anatomy and oedema. Rupture of the abscess with subsequent aspiration of pus can occur during intubation ${ }^{[17]}$.

Tracheostomy can be performed if orotracheal intubation is not possible ${ }^{[18]}$. Based on the findings of previous authors, who have reported on non tuberculous retropharyngeal abscesses in children, majority of cases responded to intravenous antibiotics exclusively. This can be effectively predicted radiologically as published in the Société française d'ORL guidelines, when the smallest diameter of the collection is less than $15 \mathrm{~mm}^{[9]}$.

Failure of response to medical treatment after 72 hours, or presence of airway compromise, would necessitate surgical drainage ${ }^{[9]}$. A similar case to ours reported a child who was successfully treated with needle aspiration, however the degree of airway compromise in this case was possibly not as severe, negating the need for more invasive and comprehensive evacuation of the collection ${ }^{[19]}$.

If the abscess is causing respiratory compromise, emergency drainage of the abscess should be considered once the airway is secure.

Where the airway is compromised, apart from securing a safe airway, surgical decompression is necessary ${ }^{[20]}$. A retropharyngeal abscess can be drained safely either by intraoral or transcervical approach ${ }^{[6,7]}$. Therapeutic aspiration has been successful and repeated as necessary ${ }^{[21]}$. Early referral to a respiratory physician and prompt initiation of anti-tuberculous chemotherapy is advised ${ }^{[1,2]}$. Pulmonary and extra-pulmonary tuberculosis is treated with the same regime, which is 6 months of isoniazid and rifampicin, with the addition of pyrazinamide and ethambutol in the first 2 months. For extra-pulmonary tuberculosis with bone involvement or TB meningitis, treatment of 9-12 months is recommended ${ }^{[1]}$. Prior to commencement of anti-tuberculous treatment, opthalmic assessment is required, as ethambutol can cause optic neuritis ${ }^{[1]}$. In one of the reported cases, ethambutol was stopped after 1 month, as it was difficult to evaluate opthalmic problems in a 5-week-old boy.

\section{CONCLUSION}

This is rare case of tuberculous retropharyngeal abscess presenting with an insidious and progressive upper airway obstruction. It highlights the importance of always considering tuberculous infection as a possible cause, particularly in endemic countries. Diagnosis is difficult especially in chronic retropharyngeal cold abscesses, when the presenting symptoms may be gradual, non specific or often misleading. Therefore, appropriate tuberculosis cultures should always be taken alongside bacterial cultures, allowing targeted antimicrobial therapy. Management of the airway should always be a priority, regardless of the diagnosis.

\section{ACKNOWLEDGEMENTS}

I would like to thank the editors and reviewers in charge of Egyptian Journal of Ear, Nose, Throat and 
Allied Sciences for giving us the opportunity for sharing our case and we hope it will be beneficial to the readers. We would also like to acknowledge our university and hospital involved as well as the patient and family members for providing us the time and permission to write this report.

\section{CONFLICT OF INTEREST}

No conflict of interest

\section{REFERENCES}

1. World Health Organization, Stop TB Initiative (World Health Organization). Treatment of tuberculosis: guidelines. World Health Organization; 2010.

2. Management of tuberculosis, Clinical Practice Guideline, Ministry of Health Malaysia 2012

3. Kothari PR, Kumar T, Jiwane A, Kulkarni B, Paul $\mathrm{S}$. An unusual cause of stridor: retropharyngeal cold abscess. Pediatric surgery international. 2002 Mar 1;18(2-3):165-7.

4. Christoforidou A, Metallidis S, Kollaras $\mathrm{P}$, Agathangelidis A, Nikolaidis P, Vital V, Markou $\mathrm{K}$. Tuberculous retropharyngeal abscess as a cause of oropharyngeal dysphagia. American journal of otolaryngology. 2012 Mar 1;33(2):272-4.

5. Kamath MP, Bhojwani KM, Kamath SU, Mahabala C, Agarwal S. Tuberculous retropharyngeal abscess. Ear, Nose and Throat Journal. 2007 Apr 1;86(4):236-8.

6. Abdul Fattah AW, Marina MB, Mazita A, Muhammad Razif MY, Primuharsa Putra SHA. Retropharyngeal abscess: Seven-year review- Clinical Presentation, Diagnosis and Management. Medicine \& Health 2009;4(2):8490.

7. Patil C, Kharat R, Deshmukh P, Singhal S, D'Souza B. Tuberculous retropharyngeal abscess without cervical spine TB. Asian Pacific journal of tropical medicine. 2011 Mar 1;4(3):251-2.

8. Page NC, Bauer EM, Lieu JE. Clinical features and treatment of retropharyngeal abscess in children. Otolaryngology-Head and Neck Surgery. 2008 Mar;138(3):300-6.
9. Martin CA, Gabrillargues J, Louvrier C, Saroul N, Mom T, Gilain L. Contribution of CT scan and CT-guided aspiration in the management of retropharyngeal abscess in children based on a series of 18 cases. European annals of otorhinolaryngology, head and neck diseases. 2014 Nov 1;131(5):277-82

10. Ravindranath $\mathrm{T}$, Janakiraman $\mathrm{N}$, Harris $\mathrm{V}$. Computed tomography in diagnosing retropharyngeal abscess in children. Clinical pediatrics. 1993 Apr;32(4):242-4.

11. Marwah A, Sethi GK, Marwah P. A large retropharyngeal abscess following spinal tuberculosis presenting as life threatening stridor in a child. JIACM. 2011;12(3):223-4.

12. Deepti BS, Munireddy M, Kamath S, Chakrabarti D. Cervical spine tuberculosis and airway compromise. Canadian Journal of Anesthesia/ Journal canadien d'anesthésie. 2016 Jun $1 ; 63(6): 768-9$.

13. Hugar BS, Chandra YG, Babu PS, Jayanth SH, Vinay J. Fatal case of retropharyngeal abscess associated with Pott's disease. Journal of forensic and legal medicine. 2013 Aug 1;20(6):567-9.

14. Idris SK, Abdulkadir AY. Tuberculous retropharyngeal abscess with posterior mediastinal extension and quadriplegia in a 13-year-old Nigerian girl. International Journal of Pediatric Otorhinolaryngology Extra. 2010 Sep 1;5(3):118-20.

15. Shin JH, Sung SI, Kim JK, Jung JM, Kim ES, Choi SH, Kim YJ, Ahn KM, Chang YS, Park WS. Retropharyngeal abscess coinfected with Staphylococcus aureus and Mycobacterium tuberculosis after rhinoviral infection in a 1-monthold infant. Korean journal of pediatrics. $2013 \mathrm{Feb}$ 1;56(2):86-9.

16. Zafereo Jr ME, Pereira KD. Chronic retropharyngeal abscess presenting as obstructive sleep apnea. Pediatric emergency care. 2008 Jun $1 ; 24(6): 382-4$

17. Rao MS, Raju YL, Vishwanathan PN Anaesthetic management of difficult airway due to retropharyngeal abscess. Indian journal of anaesthesia. 2010 May;54(3):246. 
18. Gharib B, Mohammadpour M, Sharifzadeh M, Mirashrafi F, Yaghmaie B, Pak N, Mehdizadeh M, Eshaghi H, Gorji M, Memarian S. A 15-Month-Old Boy With Respiratory Distress and Parapharyngeal Abscess: A Case Report. Acta Medica Iranica. 2016 Dec 1;54(12):812.

19. Yasan H, Doğru H. Kothari et al.:"An unusual cause of stridor: retropharyngeal cold abscess". Pediatric surgery international. 2003 Dec 5;19(11):749-.
20. Carroll N, Bain RJ, Tseung MH, Edwards RH. Tuberculous retropharyngeal abscess producing respiratory obstruction. Thorax. 1989 Jul 1;44(7):599-600.

21. Menon E, Baruah P. Tuberculous retropharyngeal abscess revisited. BMJ case reports. 2014 Mar 14;2014:bcr2013202514. 